\title{
Analysis of Cognitive Radio Spectrum Sensing Techniques
}

\author{
Md. Shahnawaz Shaikh \\ AITR Indore
}

\author{
Kamlesh Gupta \\ AITR Indore
}

\begin{abstract}
In wireless communication, signal in its propagation path always gets affected by noise signal. The radio link between transmitter and receiver varies randomly during signal propagation. This radio link may offer simple line-of-sight or multipath propagation of communication signal depending on channel condition and channel type. Sensing performance of cognitive radio network may also gets affected over various channels and requires to be calculated. This paper enlightens the implementation and analysis through simulation of Matched filtering, Energy detection and Cyclostationary feature detection cognitive radio spectrum sensing techniques over AWGN, Rician and Rayleigh fading channels. It also contains combined analysis of the three techniques in terms of the SNR vs decision accuracy plots over AWGN, Rician fading and Rayleigh fading channels.
\end{abstract}

\section{Keywords:}

Cognitive, Spectrum sensing, Energy detection, Matched filtering, Cyclostationary detection, Cooperative sensing

\section{INTRODUCTION}

The exponential and unpredicted growth in wireless communication demands additional bandwidth or efficient bandwidth utilization of existing spectra. Cognitive radio (CR) has emerged as a leading technology because it can intelligently sense an unused spectrum without creating any harmful interference to authorized users. Cognitive radio network is not introduced as a replacement but complement to the existing wireless communication networks. It can operate in licensed frequency bands in order to improve quality of service (QoS) to its users. Spectrum utilization and efficiency can be improved by allowing secondary or unlicensed users to access spectrum hole, unoccupied by a primary user (PU), at the right location and time. Spectrum sensing is a crucial step in cognitive radio. Therefore for the system to provide good quality of services to the secondary users, they need to have a valuable and trusted sensing techniques in order to sense idle spectrum holes in the network. Different from conventional wireless radios, CR is able to monitor and analyze existing spectrum usage with determination of its operating parameters, to effectively adapt varying radio environment. CR users are allowed to utilize licensed spectrum bands opportunistically, as long as they do not cause any unacceptable interference with licensed users. Such flexibility alleviates the crowding and congestion issue in particular spectrum bands and greatly enhances the efficiency of spectrum utilization. Consequently, CR technology has gained increasing attention and highlighted by both standards and regulatory bodies [1], [7], [8], [17].

CR can smartly sense and adapt idle spectrum in rapidly changing environment, using its transmitting parameters such as modulation, frequency, frame format etc. CR is being recognized as an intelligent technology to adapt operating parameters from changing environment rapidly and enormously [16]. The main challenges with cognitive radios are that it should not interfere with the licensed users and should release the band when required.

The objective of this paper is to implement and simulate Matched filtering, Energy detection and Cyclostationary feature detection cognitive radio spectrum sensing techniques as well as analyze the simulation results. This paper is organized as follows: Section II defines Matched filtering spectrum sensing technique. Section III presents Energy detection spectrum sensing techniques. Cyclostationary feature detection spectrum sensing technique is explained in section IV. Propagation channels are defined in section V. Decision accuracy also enlightens in section VI. Simulation results are shown in section VII with their respective inferences. Section VIII concludes this paper.

Figure 1 shows various aspects associated with spectrum sensing techniques alongwith various issues and associated challenges.

\section{MATCHED FILTERING}

The optimal way for spectrum sensing in cognitive radio is Matched filtering technique. It is popular due to its very accurate detection probability and less detection time, which maximizes SNR. Matched Filtering needs short time to achieve a certain probability of false alarm or probability of missed-detection [2, 7]. Matched filter perform coherent detection of primary user signal and works as a linear filter [3, 2, 1,5$]$. Output of the filter results the desired useful signal, while attenuating noise signals. To filter the desired signal, it requires a prior knowledge of every primary user signal, which needs a dedicated receiver for every type of primary user signal. However, it increases implementation complexity and power consumption. To detect the signals it also requires execution of various receiver algorithms at sensing unit [3, 2, 1, 6, 7, 8].

Primary user information e.g., modulation type and order, pulse shaping, packet format bandwidth and operating frequency might be pre-stored in CR memory. In matched filter operation, received signal is convolved with the filter impulse response which is time shifted and mirror version of reference signal, this terminology approaches to the correlation of signals [3, 2, 5, 8]. Optimal detector in stationary gaussian noise used as matched filter, when secondary user has knowledge about information of primary user sig- 


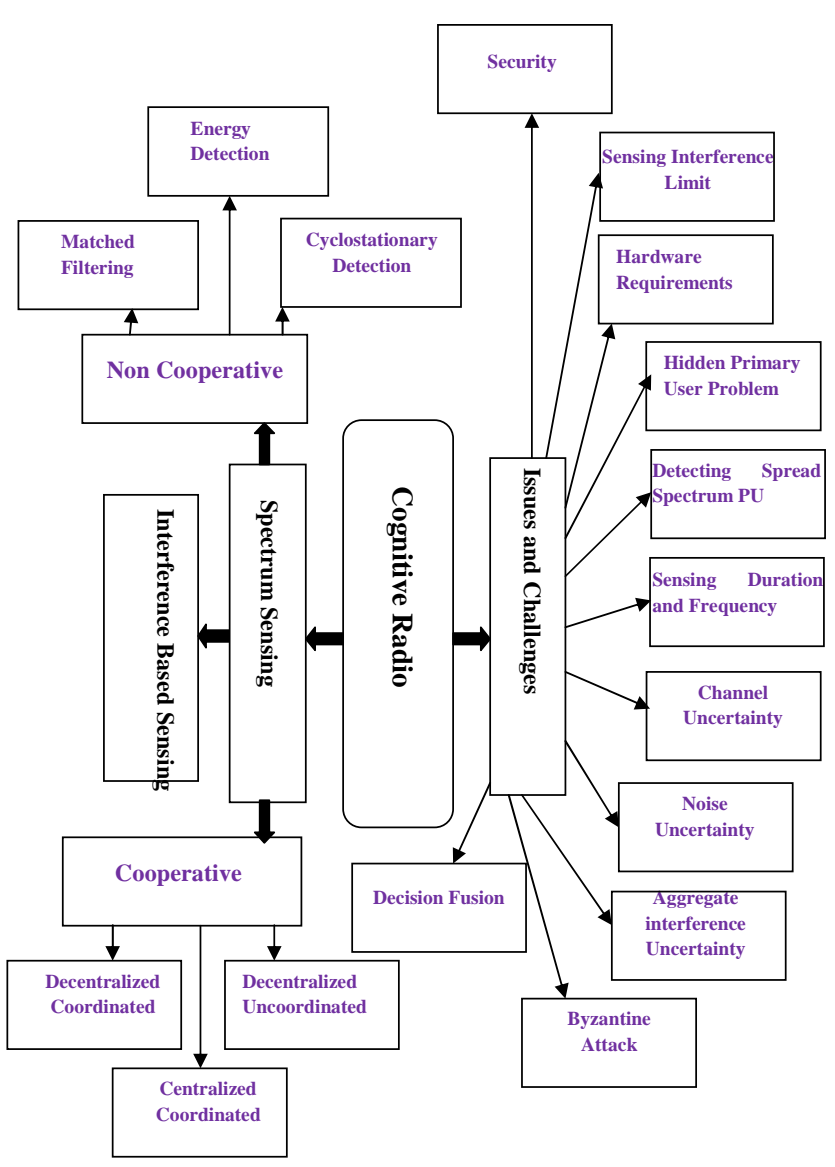

Fig. 1. Various aspects of spectrum sensing for cognitive radio

nal [8, 10]. Matched filter performs poorly in case of incomplete or inaccurate information [8,10]. The operation of matched filter detection is expressed as [3, 2, 1, 8]:

$$
Y[n]=\sum_{k=-\infty}^{+\infty} h[n-k] z[k]
$$

where $z$ is unknown signal and is convolved with the $h$, the impulse response of matched filter that is matched to the reference signal for maximizing the SNR [3, 2, 1,8$]$.

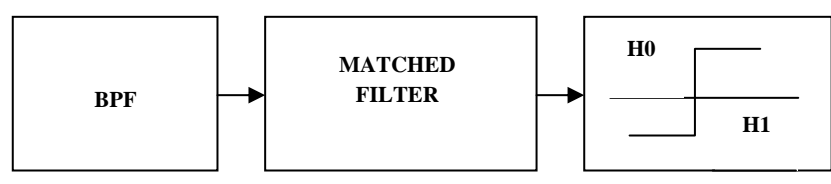

Fig. 2. Matched Filtering [2]

Figure 2 shows block diagram of Matched filtering process, which consists of a band pass filter to allow specific band spectrum. Linear matched filter performs filtering of recieved signal. Output of signal is defineds by hypothesis $H_{0}$ and $H_{1}$.

\section{ENERGY DETECTION}

Simplicity and ease of applicability of energy detection technique represents it as the most preferred approach for spectrum sensing in cognitive radio technology [16]. It is very simple and non coherent detection based sensing technique of CR [6]. The detection and comparison of signal energy introduces principle of this technique. Energy of desired spectrum calculated in this technique and compares it with threshold energy level [16]. If the signal energy lies above the threshold energy level, the respective frequency band seems to be busy. Otherwise the frequency band is supposed to be idle and could be accessed by CR users (secondary users) [4, 13, 16]. It offers low computational and implementation cost and does not require the prior knowledge about the structure or format of the primary user signal [16]. The threshold used for primary user signal detection is highly susceptible to changing noise levels. Moreover, presence of any undesired band possessing equal energy level could confuse the energy detector. Other than this, energy detector is not useful for direct sequence, frequency hopping signals and spread spectrum signals. Energy detection technique requires long time to achieve desired performance level [4, 6, 11, 13, 16]. These factors limit the performance of this technique in comparison to matched filtering and cyclostationary feature detection techniques. Spectrum sesnsing is a binary hypothesis problem described in below equation:

$$
X(t)= \begin{cases}n(t) & H_{0} \\ s(t)+n(t) & H_{1}\end{cases}
$$

where $s(t)$ represents primary user signal while noise signal received with primary user signal is denoted by $n(t) . X(t)$ define total signal received.

$H_{0}$ represents presence of noise only, while $H_{1}$ represents the presence of primary user signal [16, 17].

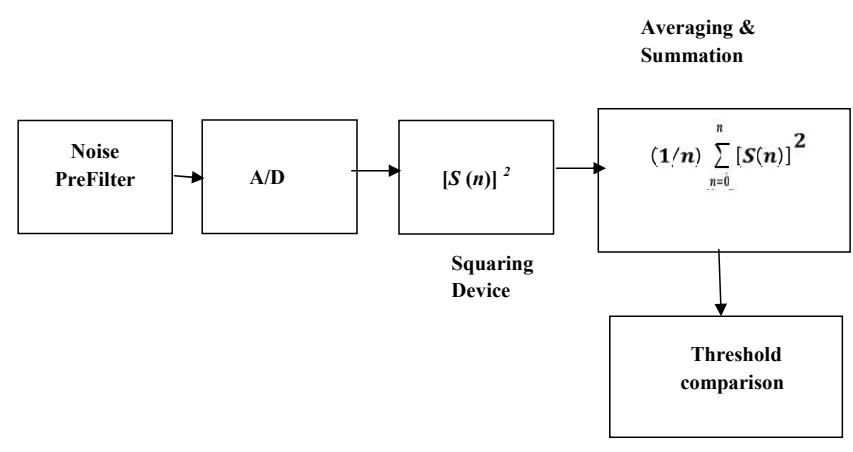

Fig. 3. Energy Detection Process [13 14, 15, 16, 17].

Figure 3 shows the block diagram of energy detector, which explains the implementation of energy detection technique. It consists of noise pre-filter which removes noise from received signal. Received analog signal is converted into its respective digital signal by A/D converter. To calculate the energy of the signal, square law device squares digital signal to calculate power within the specified window. Averaging and summation of signal, calculates signal energy over FFT window for the duration T [13, 14, 15, 16, 17, 18].

\section{CYCLOSTATIONARY FEATURE DETECTION}

Cyclostationary feature detection introduces one of the most reliable and accurate method of spectrum sensing. Signal format of 
modulated primary users signals are associated with sine wave carriers, pulse trains, repeating sequences, spreading, hoping sequences, or cyclic prefixes. It exploits periodicity as well as mean and autocorrelation of PU signals. Cyclostationary feature of primary user signal is characterized by this periodicity, which is used for detection of the signal in random radio environment. Receiver exploits cyclic features of primary user signal and performs signal detection using previously stored cyclic features of signal. Frequency and phase synchronization of signal is not required at the receiver end but prior knowledge of primary user signal is necessary. This technique performs better than energy detection method in low SNR regions. Also, noise uncertainties do not affect its performance. However, this complex two dimensional technique requires long observation time, high sampling rate and higher computational complexity, which may cause sampling time error [3, $2,1,4,5,6,7,12,13]$.

As stationary random signals are based on autocorrelation functions and power spectral density, cyclostationary signal exhibits correlation between widely separated spectral components due to spectral redundancy caused by periodicity [6].

The received signal is assumed to be of following simple form

$$
X(n)=s(n)+w(n)
$$

The cyclic spectral density (CSD) function of a received signal can be calculated as

$$
S(f, \alpha)=\sum_{\tau=-\infty}^{\infty}\left(R_{Y}^{\alpha}\right)(T) e^{-2 j \pi f T}
$$

Where

$$
\left(R_{Y}^{\alpha}\right)(T)=E\left[y(n+\tau) y(n-\tau) e^{2 j \pi \alpha n}\right]
$$

is the cyclic autocorrelation function (CAF) and $\alpha$ is the cyclic frequency. When the cyclic frequency is equal to the fundamental frequency of transmitted signal $x(n)$, then the CSD function exhibits its maximum values [2, 1, 6, 7]. Cyclic frequencies are used as features for identifying transmitted PU signals. It can be assumed to be known or they can be extracted [7]. Spectral correlation function is also termed as cyclic spectrum [1,7]. Signal analysis in cyclic spectrum domain preserves phase and frequency information related to timing parameters in modulated signals. As a result, over lapping features in the power spectrum density are non overlapping features in the cyclic spectrum [6].

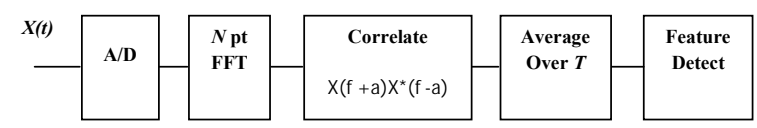

Fig. 4. Implementation of a cyclostationary feature detector

Figure 4 shows the implementation of cyclostationary feature detector, which consists an analog to digital converter whose output represented by N point FFT. The correlation among adjacent signal components introduced in the next block then calculates the average power over specified time interval.

\section{PROPAGATION CHANNEL}

The signal in its propagation path is always affected by noise signals lying in the same path. This signal path, i.e., channel may be categorized on the basis of noise distribution, scattering, dispersion and multipath propagation of signal over propagation channel. We did analysis of spectrum sensing techniques over AWGN channel, Rician fading channel and Rayleigh fading channel.

\subsection{AWGN Channel}

Additive white gaussian noise (AWGN) is a basic noise model in communication channels, which defines linear addition of wideband or white noise and gaussian distribution of amplitude. However multipath propagation, signal fading, scattering, nonlinearity or dispersion does not account for this channel.

\subsection{Rician Fading Channel}

Rician fading of propogated signal is a random model for radio propagation caused by partial cancellation of a radio signal. In rician fading cahannel a strong dominant component is present. This dominant component can be the line-of-sight wave. In Rician fading, the amplitude gain is characterized by a Rician distribution.

\subsection{Rayleigh Channel}

Rayleigh fading is the specialized model for random fading when there is no line of sight signal, and is sometimes considered as a special case of the more generalised concept of rician fading. In Rayleigh fading, the amplitude gain is characterized by a Rayleigh distribution. An assumption made in Rayleigh fading channel is that the propagating signal vary its amplitude randomly over the channel. There is no line of sight component in faded signal.

\section{DECISION ACCURACY}

Decision accuracy is a key parameter of spectrum sensing techniques, which defines quality and ability of a particular technique to work in randomly varying environment. Decision accuracy defines the extent to which the current results are close to certain reference values. Decision accuracy of spectrum sensing techniques may get affected by environmental noise, SNR of signals, occupancy of sensed spectrum etc. It may also vary over different channels such as AWGN channel, Rician fading channel and Rayleigh fading channel.

\section{SIMULATION RESULTS}

Implementation and simulation of cognitive radio spectrum sensing techniques give various important results, which show their performance over varying SNR as well as over AWGN, Rician fading and Rayleigh fading channels. Sinusoidal carrier signals in frequency range of $20 \mathrm{M} \mathrm{Hz}$ to $40 \mathrm{M} \mathrm{Hz}$ is defined for each of 20 users and calculate signal power and noise power. Modulate the signal using BPSK modulation before passing it through channel. At output of channel these modulated signals are contained noise signal also. So the spectrum sensing technique is applied on noise affected signals. Decision accuracy of each technique is calculated by assuming $70 \%$ occupancy of sensed frequency band.

\subsection{Matched Filtering}

Figure 5 shows sensing result of Matched filtering technique. This plot represents status of all 20 users (primary users), either these are present or not at their respective frequency band.

Figure 6 represents decision accuracy of matched filtering technique over AWGN, Rician fading channel and Rayleigh fading channel with respect to varying signal to noise ratio. Plot indicates decision accuracy of matched filtering technique increases with increase in SNR. This technique performs better over Rician fading 


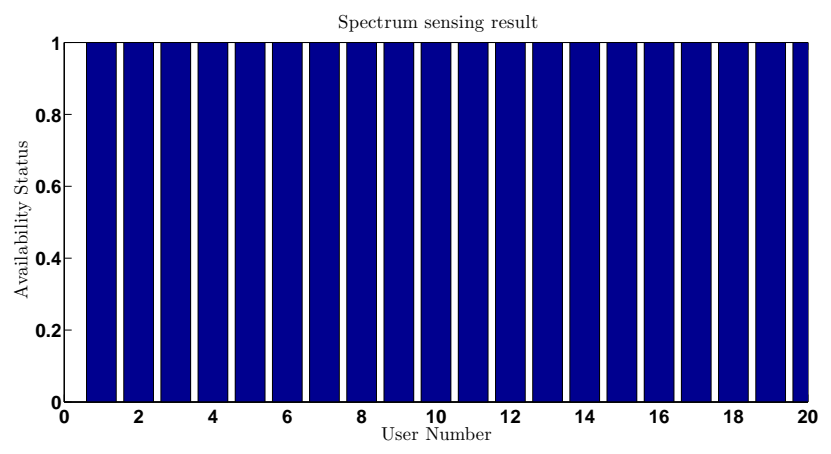

Fig. 5. Spectrum sensing decision of matched filtering technique.

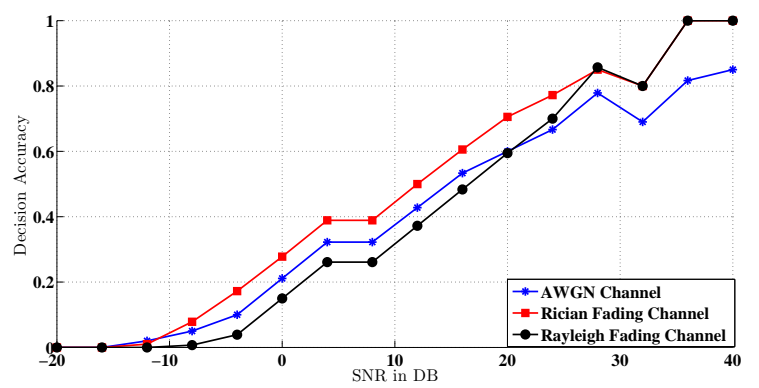

Fig. 6. SNR Vs decision accuracy for channel sensing with rectangular window for matched filtering technique.

channel while the performance over AWGN and Rayleigh fading channel also close to Rician fading channel.

\subsection{Energy Detection}

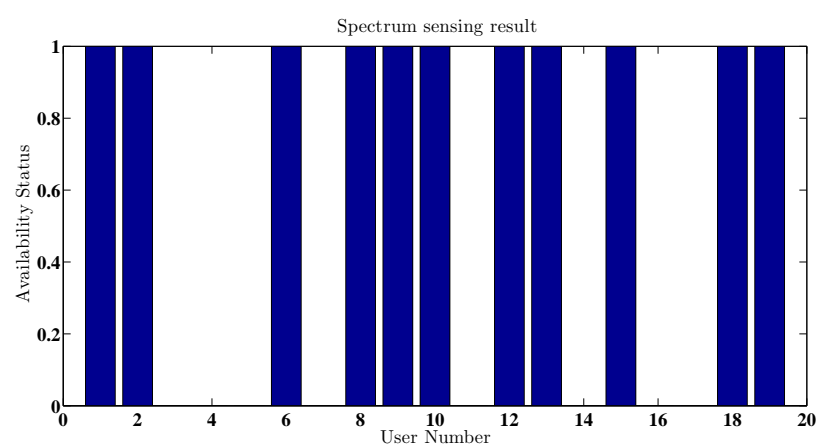

Fig. 7. Spectrum sensing decision of energy detection technique

Figure 7 shows spectrum sensing simulation result of energy detection technique. This plot represents status of all 20 users, either these are present or not at their respective frequency band.

Figure 8 represents decision accuracy of energy detection technique over AWGN, rician fading channel and rayleigh fading channel with respect to varying signal to noise ratio. Plot indicates decision accuracy of energy detection technique increases with respect to increase in SNR over both AWGN and rician fading channel,

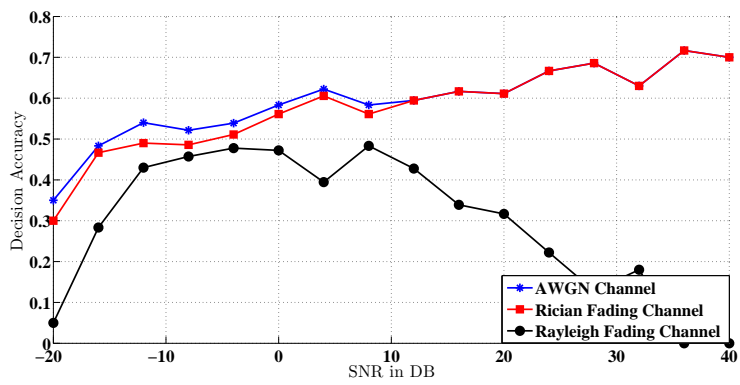

Fig. 8. SNR Vs Decision accuracy for channel sensing with rectangular window for Energy detection technique.

however its performance decreases with increase in positive SNR values over rayleigh fading channel. This technique performs better for low SNR values over all the three channels.

\subsection{Cyclostationary Feature Detection}

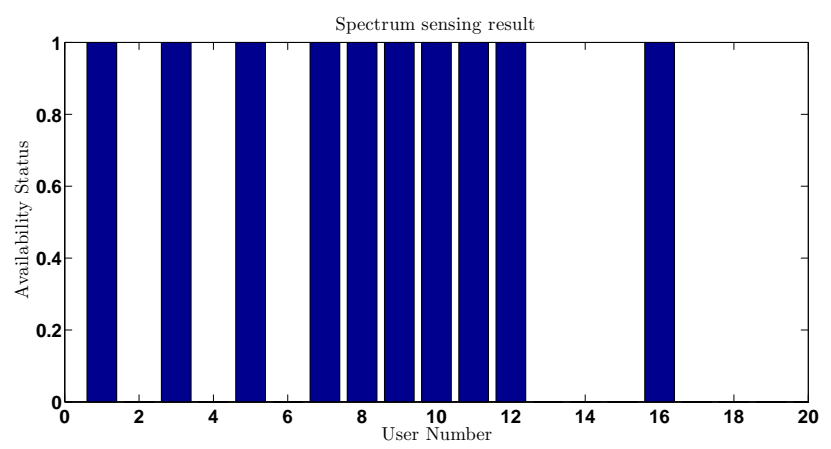

Fig. 9. Primary user signal availability status sensed through cyclostationary feature detection technique

Figure 9 shows user availability after sensing is done through cyclostationary feature detection sensing at desire band. This plot represents status of all primary users, either they are present or not at their respective frequency bands.

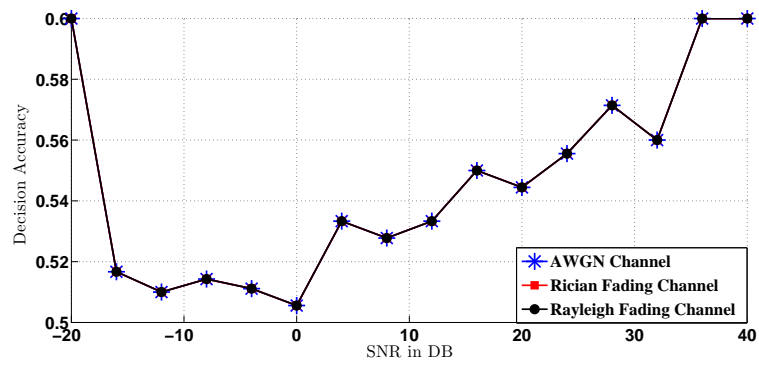

Fig. 10. SNR Vs Decision accuracy for channel sensing with rectangular window for cyclostationary feature detection technique

Figure 10 represents graph between SNR and Decision Accuracy for channel sensing with rectangular window, which indicate decision accuracy of Cyclostationary feature detection spectrum sens- 
ing technique is not depends on channel type. Its performance is same over AWGN, Rician fading and Rayleigh fading channel.

\subsection{Combine Analysis}

Implementation and simulation of these three techniques results comparative analysis of their spectrum sensing performance with respect to varying SNR on common platform. This comparative analysis also done individually over AWGN, rician fading and rayleigh fading channel.

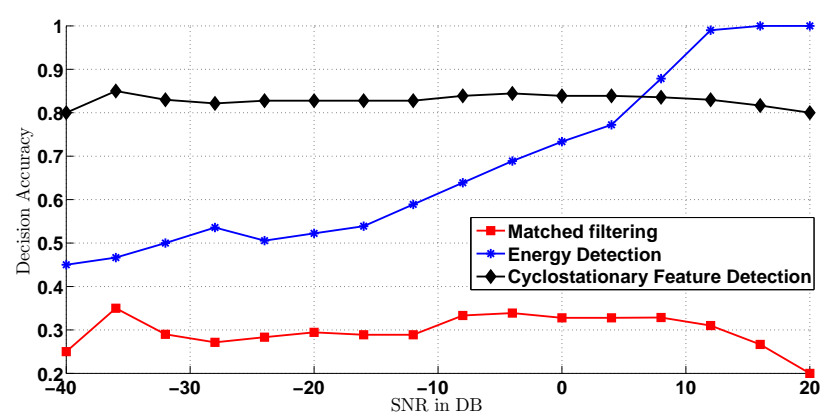

Fig. 11. Spectrum sensing decision capability for common scenario over AWGN channel

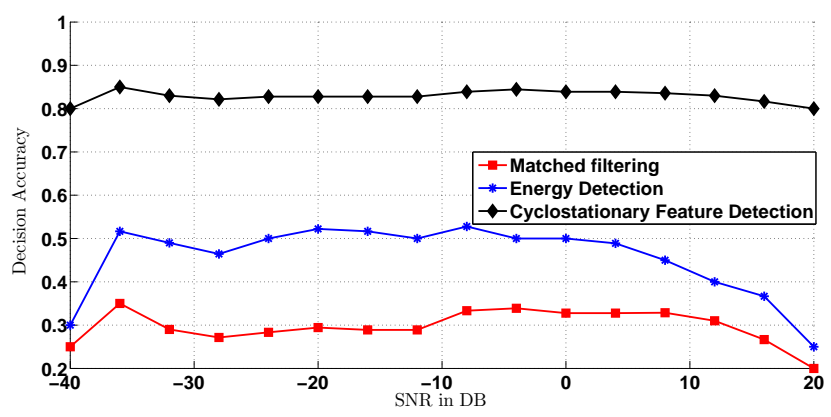

Fig. 12. Spectrum sensing decision capability for common scenario over rician fading channel

Figure 11, 12 and 13 shows comparative analysis of spectrum sensing capability of Matched filtering, Energy detection and cyclostationary feature detection techniques over AWGN, rician fading and rayleigh fading channel respectively.

In these combine plots performance of Matched filtering and cyclostationary feature detection technique over AWGN, rician fading and rayleigh fading channel has almost equal performance while Energy detection performs better over AWGN channel.

\section{CONCLUSION}

This paper investigated different spectrum sensing techniques in cognitive radio network. Cognitive Radio (CR) has emerged as a leading technology because it can intelligently sense an unused spectrum without creating any harmful interference to authorized users. It is a novel approach to fulfill increasing demand of bandwidth for effective and healthy communication. Varying SNR in radio environment may affect performance of spectrum sensing techniques. Spectrum sensing is a multifaceted problem demanding coordinated efforts of the regulatory and technical sides. In this paper a comparative analysis of spectrum sensing capability of matched filtering, Energy detection and cyclostationary feature detection technique was carried out in terms of decision accuracy vs SNR under varying channel conditions including AWGN, Rician fading, Rayleigh fading, and useful inferences were drawn.

\section{REFERENCES}

[1] V. Stoianovici, V. Popescu, M. Murroni, "A survey on spectrum sensing techniques for cognitive radio," Bulletin of the Transilvania University of Brasov Vol. 15 (50) 2008.

[2] Anita Garhwal and Partha Pratim Bhattacharya, "A survey on spectrum sensing techniques in cognitive radio," International Journal of Computer Science Communication Networks, Vol 1(2), 196-206.

[3] Sajjad Ahmad Ghauri, I M Qureshi, M. Farhan Sohail, SherazAlam, M. Anas Ashraf, "Spectrum sensing for cognitive radio networks over fading channels," International Journal of Computer and Electronics Research Vol. 2, Issue 1, February 2013.

[4] Anirudh M. Rao, B. R. Karthikeyan, Dipayan mazumdar, Govind R. Kadambi,"Energy detection technique for spectrum sensing in cognitive radio," SASTECH Vol. 9, Issue 1, April 2010

[5] Mahmood A. Abdulsattar and Zahir A. Hussein, "Energy detection technique for Spectrum sensing in cognitive Radio," International Journal of Computer Networks Communications (IJCNC) Vol.4, No.5, September 2012.

[6] Danijela Cabric, Shridhar Mubaraq Mishra, Robert W. Brodersen Berkeley Wireless Research Center, University of California, Berkeley IEEE Paper, "Implementation issues in spectrum sensing for cognitive radios," in Proc. the 38th. Asilomar Conference on Signals, Systems and Computers, year 2004, pages 772-776.

[7] Tevfik Yucek and Huseyin Arslan, "A survey of spectrum sensing algorithms for cognitive radio applications," IEEE communications surveys tutorials, Vol. 11, no. 1, first quarter 2009.

[8] Mansi Subhedar1 and Gajanan Birajdar, "Spectrum sensing techniques in cognitive radio networks: a survey," International Journal of Next-Generation Networks (IJNGN) Vol.3, No.2, June 2011, DOI : 10.5121/ijngn.2011.3203 37.

[9] A. Rahim Biswas, Tuncer Can Aysal, Sithamparanathan Kandeepan, Dzmitry Kliazovich, Radoslaw Piesiewicz, "Cooperative shared spectrum sensing for dynamic cognitive radio networks," Broadband and Wireless Group, Create-Net International Research Centre, Trento, Italy, EUWB (FP7-ICT215669).

[10] Nishant Dev Khaira, Prateek Bhadauria, "Cooperative spectrum sensing and detection efficiency in cognitive radio network," International Journal of Electronics and Computer Science Engineering ISSN-2277-1956.

[11] Amir Ghasemi, Communications Research Centre Canada and University of Toronto Elvino S. Sousa, University of Toronto, "Spectrum sensing in cognitive radio networks: Requirements, Challenges and Design Trade-offs," IEEE Communications Magazine April 2008. 
[12] Paul D. Sutton, Member IEEE, Keith E. Nolan, Member IEEE and Linda E. Doyle, Member IEEE, "Cyclostationary signatures in practical cognitive radio applications," IEEE journal on selected areas in communications, Vol. 26, no. 1, January 2008.

[13] Dong-Chan Oh and Yong-Hwan Lee, "Energy detection based spectrum sensing for sensing error minimization in cognitive radio networks," International Journal of Communication Networks and Information Security (IJCNIS) Vol. 1, No. 1, April 2009.

[14] Komal Arora, Ankush Kansal, Kulbir Singh, "Comparison of energy detection based spectrum sensing methods over fading channels in cognitive radio signal processing," An International Journal (SPIJ), Vol (5) : Issue (2) : 201144.

[15] Md. Shamim Hossain, Md. Ibrahim Abdullah, Mohammad Alamgir Hossain, "Energy detection performance of spectrum sensing in cognitive radio," I.J. Information Technology and Computer Science, 2012, 11, 11-17 Published Online October 2012 in MECS (http://www.mecs-press.org/) DOI: 10.5815/ijitcs.2012.11.02.

[16] Miguel Lpez-Bentez and Fernando Casadevall, "Improved energy detection spectrum sensing for cognitive radio," Thsis paper published in IET communication publication, IET Communications(2012), 6(8):785.

[17] Saqib Saleem and Khurram Shahzad, "Performance evaluation of energy detection based spectrum sensing technique for wireless channel," International journal of multidisciplinary sciences and engineering, Vol. 3, no. 5, may 2012.

[18] Zhiqiang Bao, Bin Wu, Pin-Han Ho and Xiang Ling, "Adaptive threshold control for energy detection based spectrum sensing in cognitive radio networks," This full text paper was peer reviewed at the direction of IEEE Communications Society subject matter experts for publication in the IEEE Globecom 2011 proceedings.

[19] S.Shobana, R.Saravanan, R.Muthaiah, "Matched filter based spectrum sensing on cognitive radio for OFDM WLANs," International Journal of Engineering and Technology (IJET), Vol. 5 Issue 1, p142, February 2013. 


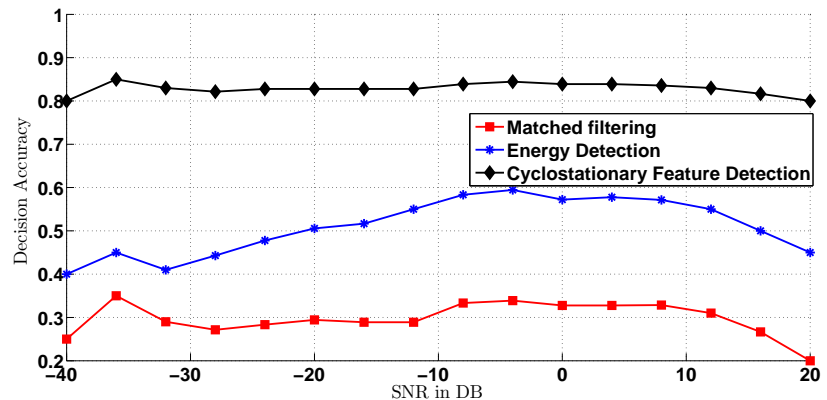

Fig. 13. Spectrum sensing decision capability for common scenario over rayleigh fading channel 